\title{
Incidence and Mortality of Malignant Melanoma in Relation to Dermatologist Density in Bavaria
}

\author{
Konstantin Drexler · Hans Drexler · Edward K. Geissler • \\ Mark Berneburg · Sebastian Haferkamp · Christian Apfelbacher
}

Received: February 18, 2021 / Accepted: September 3, 2021 / Published online: October 1, 2021

(c) The Author(s) 2021, corrected publication 2022

\begin{abstract}
Introduction: Malignant melanoma is an aggressive skin tumor with a good prognosis when treated in an early tumor stage, but has a poor prognosis with distant metastases. The incidence of malignant melanoma has increased continuously over the last decades, with little change in mortality. One explanation for this is that melanomas are increasingly
\end{abstract}

Sebastian Haferkamp and Christian Apfelbacher contributed equally.

K. Drexler $(\bowtie) \cdot$ M. Berneburg · S. Haferkamp Universitätsklinikum Regensburg, Klinik und Poliklinik für Dermatologie, Regensburg, Germany e-mail: Konstantin.drexler@ukr.de

H. Drexler

Institut und Poliklinik für Arbeits-, Sozial- und Umweltmedizin, Friedrich-Alexander-Universität

Erlangen-Nürnberg, Erlangen, Germany

E. K. Geissler

Universitätsklinikum Regensburg, Experimentelle

Chirurgie, Regensburg, Germany

E. K. Geissler

Division of Personalized Tumor Therapy,

Fraunhofer Institute for Experimental Medicine and

Toxicology, Regensburg, Germany

C. Apfelbacher

Institut für Sozialmedizin und

Gesundheitssystemforschung (ISMG), Otto von

Guericke Universität Magdeburg, Magdeburg,

Germany detected in early stages, especially after the establishment of statutory skin cancer screening in 2008, which allows a free skin examination every 2 years for people older than 35 years.

Methods: In this study incidence and mortality of malignant melanoma were correlated with the dermatologist density in Bavarian administrative regions. In addition, the incidence data were compared before and after the introduction of statutory skin cancer screening.

Results: There was a significant correlation between the incidence of malignant melanoma and dermatologist density $(r=0.258$, $p=0.044)$, but no correlation between mortality and dermatologist density $(r=0.201$, $p=0.121$ ). Similarly, the increase of malignant melanoma incidence following the introduction of statutory skin cancer screening in 2008 was independent of dermatologist density $(r=0.021, p=0.873)$.

Conclusion: The dermatologist density in Bavaria correlates positively with the incidence of malignant melanoma. Despite an increased incidence, mortality was not elevated in the respective administrative regions.

Keywords: Melanoma; Incidence; Mortality; Dermatologist density; Statutory skin cancer screening 
Key Summary Points

Dermatologist density and incidence of malignant melanoma differ a lot according to administrative area

The incidence of malignant melanoma correlates with dermatologist density

There is no correlation between the mortality of malignant melanoma and dermatologist density

The increase of incidence of malignant melanoma after introduction of statutory skin cancer screening in 2008 was independent of dermatologist density

\section{INTRODUCTION}

Malignant melanoma is an aggressive skin tumor that is responsible for over $90 \%$ of deaths due to skin cancer $[1,2]$. While detection and treatment at an early tumor stage is associated with a very good prognosis, treatment of metastatic disease is complex and the prognosis is substantially worse. Early detection and treatment is therefore a decisive factor in the medical care of malignant melanoma [3]. Germany has implemented statutory skin cancer screening since 2008, which can be performed by dermatologists as well as by general practitioners, internists, and physicians [4].

The incidence of malignant melanoma has increased continuously over the last decades in Germany, including Bavaria [5]. Reasons for this are an increasing life expectancy and changed leisure behavior with increased UV exposure. Since mortality does not appear to be increasing in pace with incidence, mortality has remained relatively stable $[5,6]$. This uneven shift could be explained by early diagnosis due to the introduction of statutory skin cancer screening in Germany as of July 1,2008 , with free regular early detection examinations available to healthy patients aged 35 years and older [4]. Furthermore, modern drugs such as checkpoint inhibitors and targeted therapy with BRAF/MEK inhibitors can prolong patient survival in cases of metastatic malignant melanoma. Skin cancer screening is performed in $81 \%$ of cases by dermatologists [7] and primary excision of malignant melanomas is performed by dermatologists in $83.6 \%$ of cases; $11.1 \%$ of excisions are performed by surgeons, $3.0 \%$ by oral maxillofacial surgeons, and single excisions by other specialists [8]. So access to skin cancer screening seems mostly dependent on the possibility to get an appointment with a dermatologist and this is easier in areas with a higher dermatologist density.

According to information given by the scientific service of the German Bundestag (the German parliament), there are 6057 practicing dermatologists in Germany. Since clear regional differences in dermatologist density have been reported [9], the question arises whether there is a correlation between this density and incidence or mortality of malignant melanoma. This study aimed to determine if there is a relationship between the dermatologist density (availability of skin cancer screening) and the number of newly diagnosed melanomas and mortality in Bavaria.

\section{METHODS}

\section{Data}

Data on the incidence and mortality of malignant melanoma, broken down by individual Bavarian administrative areas, were obtained from the Bavarian Cancer Registry [10]. The incidence and mortality values presented are aggregated for every year. The number of dermatologists per administrative area was provided by the central member administration of the Bavarian Medical Association. From this, the dermatologist density per administrative area was calculated (population figures according to the "Bayerischen Landesamt für Statistik", as of December 31, 2017 [11]). Since data available from the Bavarian Medical Association central member administration provides practicing dermatologist numbers in areas which include several different administrative areas, the data on incidence and mortality 
have been combined here to enable a correlation calculation. After introduction of the statutory skin cancer screening in Germany on July 1, 2008, we studied the average incidence from 2002 to 2008, versus the average incidence from 2009 to 2019 , in relation to respective dermatologist densities in those time frames. All data used and analyzed are not identifiable.

\section{Creation of Maps and Box Plots}

The map used was obtained from the D-maps homepage [12]. The software GNU Image Manipulation Program (GIMP) was used to process the maps. For clear presentation, terciles were formed from the collected data on dermatologist density and incidence values, with these being displayed in color. In addition, the change in melanoma incidence was calculated as the difference between the average incidence from 2002 to 2008 and the average incidence between 2009 and 2019; these results are displayed as a map.

\section{Calculation of Correlations}

The statistical software package IBM SPSS Statistics 25 (IBM SPSS, Chicago, IL, USA) was used to calculate Spearman correlation coefficients and to create point-scatter plots and box plots. Statistically significant results were assumed for $p$ values below 0.05 . Calculated $r$ values were interpreted according to Cohen, assuming a strong effect for values above 0.8 , a medium effect for values between 0.5 and 0.8 , and a weak effect for values between 0.2 and 0.5 .

The correlations of incidence from 2002 to 2019, mortality from 2002 to 2019 , and the increase in incidence after the introduction of statutory skin cancer screening in 2008 were calculated for the dermatologist density per administrative area. In addition, a correlation calculation was performed between incidence from 2002 to 2019 and mortality from 2002 to 2019 , as well as the increase in incidence after 2008; a box plot was created to illustrate the relationship between dermatologist density and incidence between 2002 and 2019. The terciles of dermatologist density were also used here.
The Student $t$ test was used to test for statistically significant differences between groups.

\section{RESULTS}

\section{Incidence of Malignant Melanoma in Relation to Dermatologist Density}

The incidence of malignant melanoma between 2002 and 2019 in Bavaria was 18.2/100,000 inhabitants (range 11.85-27.4/100,000 inhabitants, per administrative area). The dermatologist density in Bavaria averaged 6.47 per 100,000 inhabitants (range 1.46-16.98/100,000 inhabitants, per administrative area). Significant differences in the incidence, as well as the dermatologist density in the individual administrative areas, were observed (Fig. 1A, B). When comparing terciles of dermatologist density to malignant melanoma incidence between 2002 and 2019, a statistically significant difference was observed between the upper and lower tercile ( $p=0.022$, Fig. 1C). Furthermore, the dermatologist density correlated significantly with the incidence of melanoma $(r=0.258, p=0.044 ;$ Fig. 1D). While this is a weak correlation, it supports the finding in Fig. 1C.

\section{Increase in Incidence Following the Introduction of Statutory Skin Cancer Screening}

Considering the pre- versus post-statute periods, the incidence of malignant melanoma increased from $15.8 / 100,000$ inhabitants (2002-2008) to 19.5/100,000 inhabitants (2009-2019) across Bavaria. Malignant melanoma incidence showed regionally varying changes after the introduction of statutory skin cancer screening (Fig. 2A, $B)$. In addition, while there was an increase in incidence in most administrative areas, there were decreases in incidence in some districts (Fig. 2C). No correlation was found between changes in incidence and dermatologist density per administrative area $(r=0.021, p=0.873$; Fig. 2D). Thus, the increase in incidence after 2008, considered per individual administrative area, appears to be independent of the number of dermatologists practicing there. 
Dermatologist density

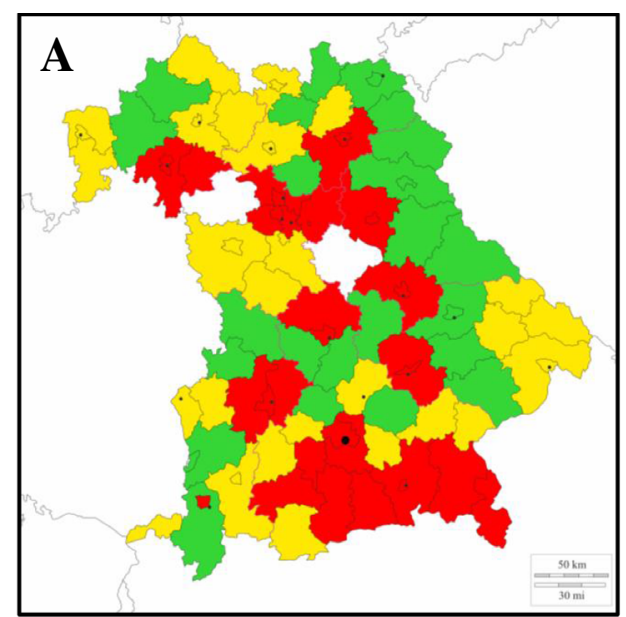

$3.51-1.46$

$5.55-3.55$

$16.98-5.67$

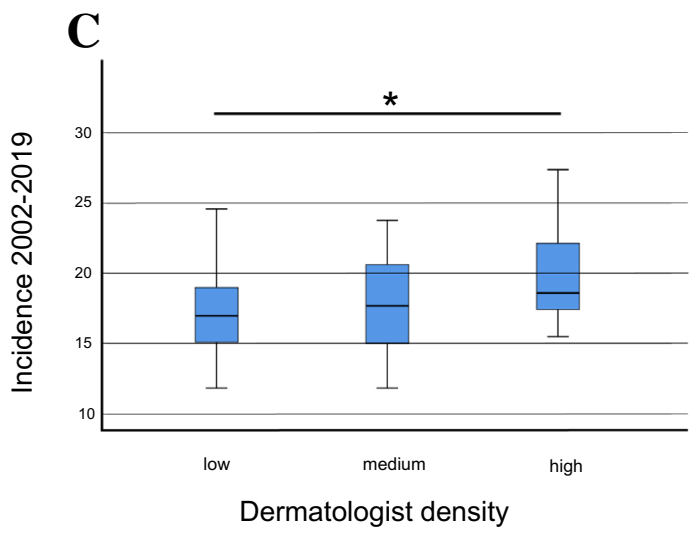

Fig. 1 Correlation of the world-standardized average incidence with dermatologist density. A Dermatologist density per administrative area in Bavaria. Administrative areas with high (red), medium (yellow), and low (green) dermatologist density. B Average, age-standardized incidence per administrative area in Bavaria. Administrative areas with high (red), medium (yellow), and low (green) incidence. All values shown refer to 100,000 inhabitants. For the white districts, no complete data were available. C Box plot showing the density of dermatologists

\section{Mortality of Malignant Melanoma in Relation to Dermatologist Density and Incidence}

The mortality rate of patients with malignant melanoma in Bavaria between 2002 and 2019

\section{Incidence 2002-2019}

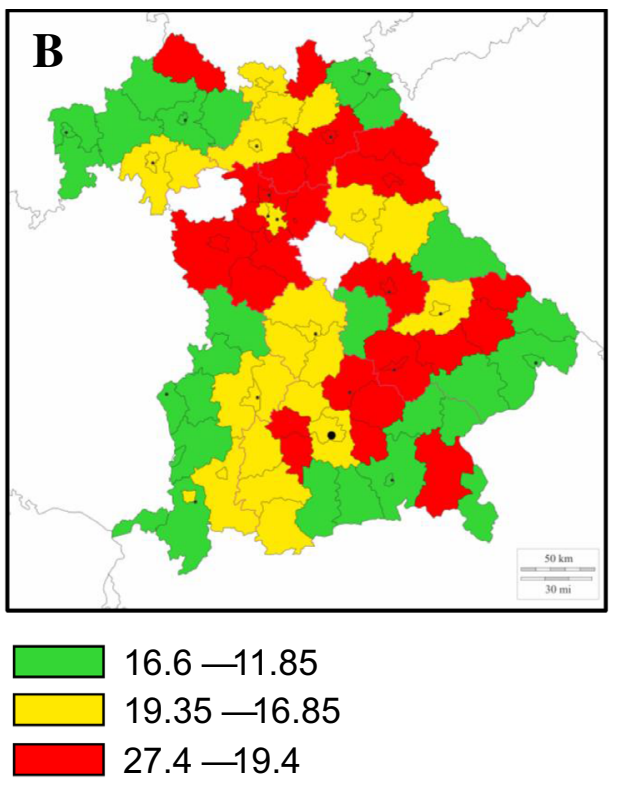

D

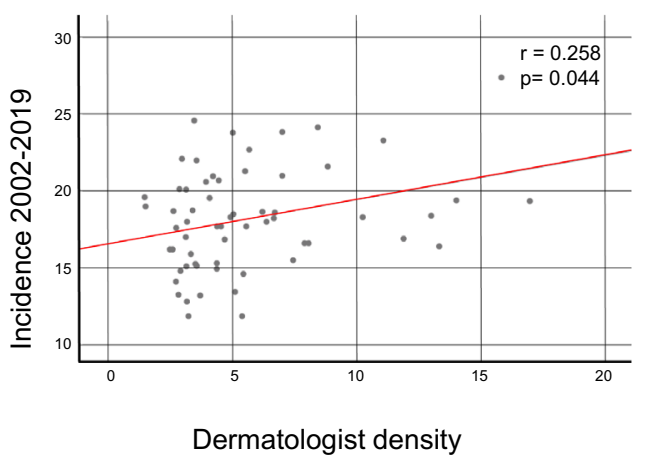

in terciles compared to the incidence between 2002 and 2019. The upper tercile is statistically significant compared to the lower tercile $(p=0.022)$. Means of incidences: low density $17.19 / 100,000$ inhabitants, medium density $17.71 / 100,000$ inhabitants, high density 19.75/100,000 inhabitants. D Scatter plot showing the correlation of dermatologist density and the incidence of malignant melanoma between 2002 and 2019 (calculation of the correlation coefficient according to Spearman, $r=0.258, p=0.044$ )

was 2.4/100,000 inhabitants. When we looked at mortality in the administrative areas, values between 3.1/100,000 and 1.55/100,000 inhabitants were seen. In relation to the dermatologist density, no statistically significant correlation was found $(r=0.201, p=0.121 ;$ Fig. $2 \mathrm{E})$. In 
addition, no statistically significant correlation was observed between incidence and mortality in the different administrative areas $(r=-0.063, p=0.632)$. Interestingly the increase in malignant melanoma incidence showed a statistically significant negative correlation with mortality $(r=-0.307, p=0.016)$. Thus, our analysis indicates that the mortality of malignant melanoma is independent of dermatologist density per administrative area, but correlates negatively with the increase of incidence after 2008.

\section{DISCUSSION}

In the current study we found that the incidence of malignant melanoma correlates with dermatologist density in the respective administrative area in Bavaria, Germany. Interestingly, despite an increased incidence in malignant melanoma, mortality was not elevated in the respective administrative areas. After introduction of statutory skin cancer screening in Germany, there was an increase in the incidence of malignant melanoma, which differed between the administrative areas and was independent of the dermatologist density.

Augustin et al. described an increased prevalence of non-melanocytic skin tumors in regions with an increased dermatologist density, but did not find this when considering the prevalence of malignant melanoma [13]. Our study showed that new cases are registered more frequently in those administrative areas where there is a higher dermatologist density, and thus better access to skin cancer screening. This could be related to reports that higher socioeconomic status has been associated with the risk of malignant melanoma [8]. Another confounding factor could be the migration of patients for the skin cancer screening to another administrative area, especially when the dermatologist density is low in the area in which they live. This is potentially a disturbing factor and should be investigated in further studies. Moreover, our data only refer to Bavaria; we cannot rule out that analyses of other regions in Germany could show different, possibly stronger or weaker correlations.
When considering the increase in malignant melanoma incidence after initiating statutory skin cancer screening in Germany, which is accepted and practiced by the general population [14], regional differences in the increase in incidence were initially observed. However, our data did not show a correlation between the dermatologist density and the malignant melanoma incidence increase seen after introducing statutory skin cancer screening. A possible explanation could be the performance of skin cancer screening by non-dermatologists, who are entitled to do so after special training [4]; these physicians perform 19\% of skin cancer screenings [7]. In areas with a low dermatologist density, skin cancer screenings performed by physicians from other specialties (after introduction of statutory skin cancer screening) may account for an even higher percentage, potentially distorting the data here. Notably, a limitation of our study is that we only examined the dermatologist density and not the number of screenings performed.

The increase in malignant melanoma mortality is significantly lower compared to projected values based on the increased incidence [6]. In our study, no significant correlations were found when looking at either the average standardized mortality (2002-2019) in relation to dermatologist density or in relation to the average standardized incidence (2002-2019), but a significant correlation between the increase of incidence after 2008 and mortality was seen. Minini et al. also describe an increase in the incidence of malignant melanoma for the canton of Zurich, Switzerland. This increase is in particular due to an increase in primary melanomas in an early tumor stage (vertical tumor thickness according to Breslow below $1.00 \mathrm{~mm}$ ), which are associated with a low risk of metastasis and thus a low risk of dying from this melanoma; the incidence of primary melanomas with a high risk of metastasis (vertical tumor thickness according to Breslow above $2.00 \mathrm{~mm}$ ) remained unchanged [15]. While a higher vertical tumor thickness could date from a rapid ab initio growth or delayed diagnosis, this could reasonably explain increasing incidence without increased mortality [16]. A high dermatologist density correlates with a lower 
Incidence 2002- 2008

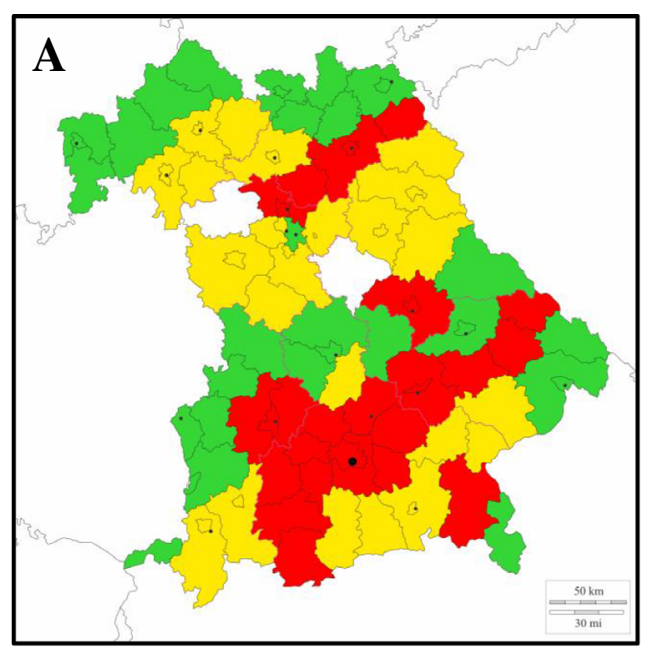

$\square$

$13.8-8.6$

$17-14$

$24.9-17.1$

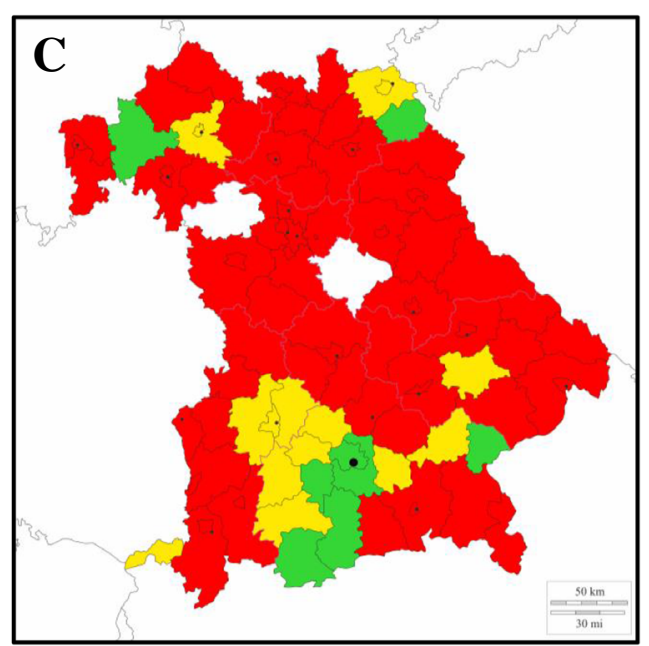

Decrease in incidence Increase in incidence 0 - 1 Increase in incidence $>1$
Incidence 2009- 2019

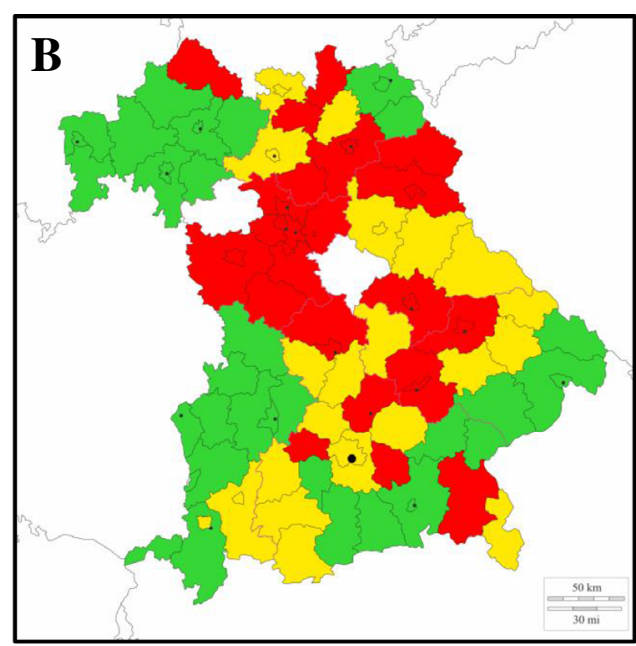

$17.4-8.6$

$21-17.5$

$32.6-21.1$
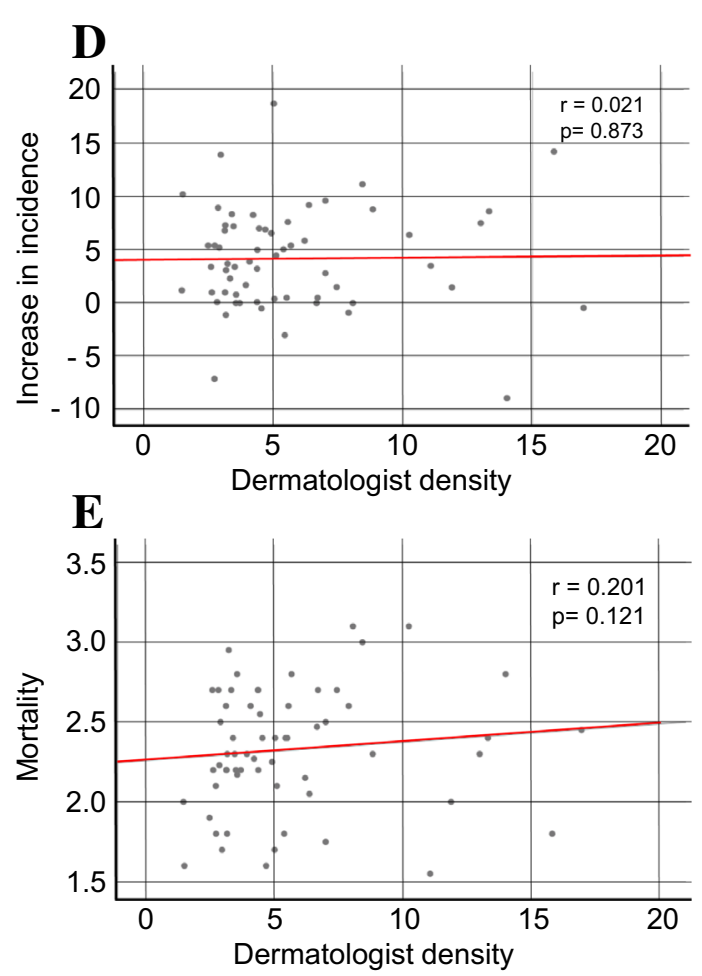
4 Fig. 2 Change in incidence after the introduction of statutory skin cancer screening. A, B Average agestandardized incidence per administrative area in Bavaria from 2002 to 2008 (A) and 2009 to 2019 (B). Administrative areas with high (red), medium (yellow), and low incidence (green). All values shown refer to 100,000 inhabitants. For the white areas, no complete data were available. C Increase in incidence in the individual administrative areas. High (red) and low (yellow) increase and decrease (green). D Scatter plot showing the correlation of dermatologist density and increase in the incidence of malignant melanoma after introduction of statutory skin cancer screening (calculation of the correlation coefficient according to Spearman, $r=0.021, p=0.873$ ). E Scatter plot showing the correlation of dermatologist density and average mortality of malignant melanoma between 2002 and 2019 (calculation of the correlation coefficient according to Spearman, $r=0.201, p=0.121$ )

thickness of primary melanoma [17]. An alternative explanation could be overdiagnosis. However, since stage of the tumor disease (including TNM classification) was not available in our data set, we cannot clarify the likelihood of this explanation. Further factoring into this discussion is that treatment of patients with metastatic malignant melanoma is mostly performed in skin tumor centers or oncological centers, which are not necessarily located in the administrative areas where the initial diagnosis was made; therefore, this issue could complicate results related to the dermatologist density of individual administrative areas. Finally, it is notable that Eide et al. described a better prognosis for patients with malignant melanoma in the USA (1988-1993) in regions with a higher dermatologist density, whereby the prognosis was defined by the ratio of mortality to incidence [18]. Also a reduction in melanoma mortality rate was shown to be associated with a higher dermatologist density [19] or a better supply of family physicians in male patients [20]. However, the dermatologist density in the USA was clearly lower compared to the dermatologist density in Bavaria, where in 2016 it was $3.4 / 100,000$ and 2009 it was 3.2/100,000 inhabitants [21].

The study carried out here was based on reliable data from the Bavarian Cancer Registry, which has been systematically recording cancer cases in Bavaria since January 1, 1998 [22]. Data used here to calculate the dermatologist density originates from the Bavarian State Medical Association, which is also reliable. However, a limitation is that the dermatologist density is a snapshot, whereas the incidence and mortality values were observed over several years. Moreover, the administrative areas and the medical district associations in Bavaria are not completely concordant, so averaging the data could be an issue of weakness.

This study shows a correlation between incidence of malignant melanoma and the dermatologist density in Bavaria, which did not correlate with increased mortality. This could be explained by the early detection of malignant melanomas, thus improving patient prognosis. This would be a highly desirable effect of the screening program for diagnosis of malignant melanoma. In addition to mortality, a disease with advanced melanoma often brings with it several accompanying symptoms, such as reduced quality of life, which require treatment [1]. Data from our study therefore support the value of continued guaranteed access to dermatologic care, where comprehensive early counseling and follow-up examinations can substantially reduce morbidity and mortality associated with the increasing incidence of metastatic malignant melanoma. Thus, our analysis indicates that the mortality of malignant melanoma is hardly correlated with both incidence and dermatologist density per administrative area and the increase of incidence is not correlated with a higher mortality.

\section{CONCLUSIONS}

The dermatologist density in Bavaria's administrative areas correlates positively with the incidence of malignant melanoma. Despite an increased incidence, mortality was not elevated in the respective administrative regions. After introduction of statutory skin cancer screening in 2008, incidence of malignant melanoma increased independent of dermatology density. 


\section{ACKNOWLEDGEMENTS}

The authors would like to thank Bavarian Medical Association and the Bavarian Cancer Registry for providing the data. The authors also thank Julia Drexler, Jan Zöllick and Florian Zeman for their support in analyzing the correlation calculations. We thank the participants of the study.

Disclosures. Konstantin Drexler is a collegiate member of the "Else-Kröner-FreseniusStiftung". Konstantin Drexler, Hans Drexler, Edward K. Geissler, Mark Berneburg, Sebastian Haferkamp and Christian Apfelbacher have nothing to disclose.

Funding. Open Access funding enabled and organized by Projekt DEAL.

Compliance with Ethics Guidelines. Data were obtained from the Bavarian Cancer Registry [10]. All data used and analyzed are not identifiable.

Authorship. All named authors meet the International Committee of Medical Journal Editors (ICMJE) criteria for authorship for this article, take responsibility for the integrity of the work as a whole, and have given their approval for this version to be published.

Authors' Contributions. Konstantin Drexler, Hans Drexler, Sebastian Haferkamp and Christian Apfelbacher contributed significantly to the conception and design of the work, the analysis, interpretation, and drafting of the most recently submitted version of the manuscript. Mark Berneburg and Edward K. Geissler contributed significantly to the interpretation and drafting of the most recently submitted version.

Prior Presentation. The data was presented at "ADO-Conference 2020" and "ADF Conference 2021".

Data Availability. The datasets generated during and/or analyzed during the current study are available from the corresponding author upon reasonable request.

Open Access. This article is licensed under a Creative Commons Attribution-NonCommercial 4.0 International License, which permits any non-commercial use, sharing, adaptation, distribution and reproduction in any medium or format, as long as you give appropriate credit to the original author(s) and the source, provide a link to the Creative Commons licence, and indicate if changes were made. The images or other third party material in this article are included in the article's Creative Commons licence, unless indicated otherwise in a credit line to the material. If material is not included in the article's Creative Commons licence and your intended use is not permitted by statutory regulation or exceeds the permitted use, you will need to obtain permission directly from the copyright holder. To view a copy of this licence, visit http://creativecommons.org/licenses/by$\mathrm{nc} / 4.0 /$.

\section{REFERENCES}

1. Leitlinienprogramm Onkologie (Deutsche Krebsgesellschaft, Deutsche Krebshilfe, AWMF): Diagnostik, Therapie und Nachsorge des Melanoms, Langversion 3.3, 2020, AWMF Registernummer: 032/024OL. http://www.leitlinienprogrammonkologie.de/leitlinien/melanom/. Accessed 2 Feb 2021.

2. Hübner J, Waldmann A, Geller AC, et al. Interval cancers after skin cancer screening: incidence, tumour characteristics and risk factors for cutaneous melanoma. Br J Cancer. 2017;116:253-9.

3. Leitlinienprogramm Onkologie (Deutsche Krebsgesellschaft, Deutsche Krebshilfe, AWMF): S3-Leitlinie Prävention von Hautkrebs, Langversion 2.1, 2021, AWMF Registernummer: 032/052OL. https://www. leitlinienprogramm-onkologie.de/leitlinien/ hautkrebs-praevention/. Accessed 2 Feb 2021.

4. Bundesministerium für Gesundheit. Bekanntmachung eines Beschlusses des Gemeinsamen Bundesausschusses über eine Änderung der Krebsfrüherkennungs-Richtlinien: Hautkrebsscreening. https://www.g-ba.de/beschluesse/516/. Accessed 2 Feb 2021. 
5. Garbe C, Keim U, Eigentler TK, et al. Time trends in incidence and mortality of cutaneous melanoma in Germany. J Eur Acad Dermatol Venereol. 2019;33: $1272-80$

6. Breitbart EW, Waldmann A, Nolte S, et al. Systematic skin cancer screening in Northern Germany. J Am Acad Dermatol. 2012;66:201-11.

7. Krensel M, Schäfer I, Zander $\mathrm{N}$, et al. Primärprävention im Rahmen des Hautkrebsscreenings. Hautarzt. 2019;70:432-7.

8. Augustin M, Anastasiadou Z, Schaarschmidt ML, et al. Versorgung des Hautkrebses in Deutschland Leistungsvolumina und -erbringer. Hautarzt. 2016;67:544-8.

9. Wissenschaftliche Dienste des Deutschen Bundestags. Anzahl der Ärzte verschiedener Fachrichtungen: Statistische Angaben zu Deutschland und ausgewählten europäischen Ländern; Aktenzeichen: WD 9 - 3000 - 053/19; 2019.

10. Melanom der Haut, C43, Inzidenz und Mortalität, alterstandardisiert, Europastandard. https://www. krebsregister-bayern.de/lgl_abfrage_d.php. Accessed Jul 7, 2021.

11. Bayerisches Landesamt für Statistik. Einwohnerzahlen am 31. Dezember 2017: Gemeinden, Kreise und Regierungsbezirke in Bayern Basis Zensus 2011 2018. Statistische Berichte - A12003 201744 - Einwohnerzahlen am 31. Dezember 2017.

12. D-maps. https://d-maps.com/carte.php?num_car= 23847\&lang=de. Accessed Mar 23, 2020.

13. Augustin J, Kis A, Sorbe C, et al. Epidemiology of skin cancer in the German population: impact of socioeconomic and geographic factors. J Eur Acad Dermatol Venereol. 2018;32:1906-13.
14. Augustin M, Stadler R, Reusch M, et al. Skin cancer screening in Germany-perception by the public. J Dtsch Dermatol Ges. 2012;10:42-9.

15. Minini R, Rohrmann $S$, Braun $R$, et al. Incidence trends and clinical-pathological characteristics of invasive cutaneous melanoma from 1980 to 2010 in the canton of Zurich, Switzerland. Melanoma Res. 2017;27:145-51.

16. Gassenmaier $\mathrm{M}$, Stec $\mathrm{T}$, Keim $\mathrm{U}$, et al. Incidence and characteristics of thick second primary melanomas: a study of the German Central Malignant Melanoma Registry. J Eur Acad Dermatol Venereol. 2019;33:63-70.

17. Moreau JF, Weinstock MA, Geller AC, et al. Individual and ecological factors associated with early detection of nodular melanoma in the United States. Melanoma Res. 2014;24:165-71.

18. Eide MJ, Weinstock MA, Clark MA. The association of physician-specialty density and melanoma prognosis in the United States, 1988 to 1993. J Am Acad Dermatol. 2009;60:51-8.

19. Aneja S, Bordeaux JS. Association of increased dermatologist density with lower melanoma mortality. Arch Dermatol. 2012;148:174-8.

20. van Durme DJ, Ullman R, Campbell RJ, et al. Effects of physician supply on melanoma incidence and mortality in Florida. South Med J. 2003;96:656-60.

21. Becker LR, Aakhus AE, Reich HC, et al. A novel alternate dosing of vismodegib for treatment of patients with advanced basal cell carcinomas. JAMA Dermatol. 2017;153:321-2.

22. Bevölkerungsbezogenes Krebsregister Bayern. Bericht des Bevölkerungsbezogenen Krebsregisters Bayern für die Jahre 1998 und 19992000. 